International Conference

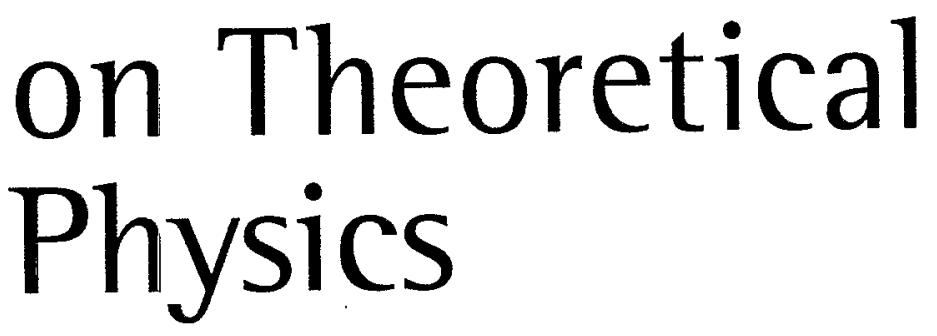

TH-2002, Paris, July 22-27, 2002

Daniel lagolnitzer Vincent Rivasseau Jean Zinn-Justin Editors 


\title{
Dynamical Response of Quasi 1D Mott Insulators
}

\author{
Fabian H.L. Essler and Alexei M. Tsvelik
}

\begin{abstract}
At low energies certain one dimensional Mott insulators can be described in terms of an exactly solvable quantum field theory, the U(1) Thirring model. Using exact results derived from integrability we determine dynamical properties like the frequency dependent optical conductivity and the single-particle Green's function. We discuss the effects of a small temperature and the effects on interchain tunneling in a model of infinitely many weakly coupled chains.
\end{abstract}

\section{Introduction}

The problem of the Mott metal-insulator transition, which is a paradigm for the importance of electron-electron interactions, has been the subject of great interest [1] since the pioneering works by Mott [2]. The intrinsic difficulty in describing the Mott transition quantitatively is that it occurs when the kinetic and potential energies are of the same order. This regime is difficult to access from either the "band" (where one diagonalizes the kinetic energy first and then takes electronelectron interactions into account perturbatively) or the "atomic" (where one diagonalizes the electron-electron interaction first and then takes the hopping into account perturbatively) limits. In recent years much progress in understanding the Mott transition has been made based on the so-called Dynamical Mean Field theory, which considers a lattice in infinitely many dimensions $D \rightarrow \infty$ (see Ref.[3] and references therein). Here we are concerned with the rather different situation, where the tunneling along one lattice direction is much larger than along all others. The resulting system can be thought of in terms of weakly coupled chains of electrons and will be referred to as a quasi one dimensional Mott insulator. When the band is half-filled and the interchain tunneling is switched off, Umklapp processes dynamically generate a spectral gap $M$ and we are dealing with an ensemble of uncoupled 1D Mott insulating chains. The same Umklapp scattering mechanism can generate gaps at any commensurate filling e.g. quarter filling, but only if the interactions are sufficiently strong. There are two questions we want to address: (i) What is the dynamical response of the uncoupled Mott-insulating chains system? and (ii) What are the effects of a weak interchain tunneling? Examples of materials that are believed to fall into the general category of quasi-1D Mott insulators are the Bechgaard salts [4] and chain cuprates like $\mathrm{SrCuO}_{2}, \mathrm{Sr}_{2} \mathrm{CuO}_{3}{ }^{1}$ or $\mathrm{PrBa}_{2} \mathrm{Cu}_{3} \mathrm{O}_{7}$. They have been found to exhibit very rich and unusual physical properties such as spin-charge separation $[5,6]$.

\footnotetext{
${ }^{1}$ More precisely, these compounds are considered to be charge-transfer insulators.
} 


\section{The Models}

The simplest models used in the description of one dimensional Mott insulators are "extended" Hubbard models of the form

$$
\hat{H}=-t \sum_{n, \sigma}\left[c_{n, \sigma}^{\dagger} c_{n+1, \sigma}+\text { h.c. }\right]+U \sum_{k} n_{k, \uparrow} n_{k, \downarrow}+\sum_{j \geq 1} V_{j} \sum_{k} n_{k} n_{k+j},
$$

where $n_{k, \sigma}=c_{k, \sigma}^{\dagger} c_{k, \sigma}$ and $n_{k}=n_{k, \uparrow}+n_{k, \downarrow}$ are electron number operators. The electron-electron interaction terms mimic the effects of a screened Coulomb interaction. Two cases are of particular interest from the point of view of application to e.g. the Bechgaard salts [4, 7]: (1) Half filling (1 electron per site) and (2) Quarter filling ( 1 electron per 2 sites). We will discuss both these cases and emphasize similarities and differences in their dynamical response.

\section{Field theory description of the low energy limit}

The field theory limit is constructed by keeping only the low-energy modes in the vicinity of the Fermi points $\pm k_{F}$. We may express the lattice electron annihilation operator in terms of the slowly varying (on the scale of the lattice spacing $a_{0}$ ) right and left moving electron fields $R(x)$ and $L(x)$

$$
c_{l, \sigma} \rightarrow \sqrt{a_{0}}\left[\exp \left(i k_{F} x\right) R_{\sigma}(x)+\exp \left(-i k_{F} x\right) L_{\sigma}(x)\right] .
$$

Here $k_{F}=\pi / 2 a_{0}$ for the half-filled band and $x=l a_{0}$. The resulting Fermion Hamiltonian can then be bosonized by standard methods [8].

\section{A. Half Filled Band}

For the half-filled case we have

$$
\begin{aligned}
& L_{\sigma}^{\dagger}(x)=\frac{\eta_{\sigma}}{\sqrt{2 \pi}} e^{i f_{\sigma} \pi / 4} \exp \left(-\frac{i}{4}\left[\beta \Phi_{c}-\frac{1}{\beta} \Theta_{c}\right]\right) \exp \left(-\frac{i f_{\sigma}}{4}\left[\Phi_{s}-\Theta_{s}\right]\right), \\
& R_{\sigma}^{\dagger}(x)=\frac{\eta_{\sigma}}{\sqrt{2 \pi}} e^{i f_{\sigma} \pi / 4} \exp \left(\frac{i}{4}\left[\beta \Phi_{c}+\frac{1}{\beta} \Theta_{c}\right]\right) \exp \left(\frac{i f_{\sigma}}{4}\left[\Phi_{s}+\Theta_{s}\right]\right),
\end{aligned}
$$

where $\eta_{a}$ are Klein factors with $\left\{\eta_{a}, \eta_{b}\right\}=2 \delta_{a b}$ and where $f_{\uparrow}=1, f_{\downarrow}=-1^{2}$. The canonical Bose fields $\Phi_{s . c}$ and their respective dual fields $\Theta_{s . c}$ are given by

$$
\Phi_{a}=\phi_{a}+\bar{\phi}_{a}, \quad \Theta_{a}=\phi_{a}-\bar{\phi}_{a}, a=s, c,
$$

where the chiral boson fields $\phi_{a}$ and $\bar{\phi}_{a}$ fulfill the following commutation relations

$$
\left[\phi_{a}(x), \bar{\phi}_{a}(y)\right]=2 \pi i, \quad a=c, s .
$$

\footnotetext{
${ }^{2}$ The phase factors in (3) have been introduced in order to ensure the standard bosonization formulas foe the staggered magnetizations.
} 
We choose a normalization such that for $|x-y| \longrightarrow 0$ the following operator product expansion holds

$$
\exp \left(i \alpha \Phi_{c}(x)\right) \exp \left(i \beta \Phi_{c}(y)\right) \longrightarrow|x-y|^{4 \alpha \beta} \exp \left(i \alpha \Phi_{c}(x)+i \beta \Phi_{c}(y)\right) .
$$

Applying the bosonization identities we obtain the following bosonic form of the low energy effective Hamiltonian

$$
\begin{aligned}
\mathcal{H} & =\mathcal{H}_{\mathrm{c}}+\mathcal{H}_{\mathrm{s}} \\
\mathcal{H}_{\mathrm{s}} & =\frac{v_{\mathrm{s}}}{16 \pi}\left[\left(\partial_{x} \Theta_{\mathrm{s}}\right)^{2}+\left(\partial_{x} \Phi_{\mathrm{s}}\right)^{2}\right], \\
\mathcal{H}_{\mathrm{c}} & =\frac{v_{\mathrm{c}}}{16 \pi}\left[\left(\partial_{x} \Theta_{\mathrm{c}}\right)^{2}+\left(\partial_{x} \Phi_{\mathrm{c}}\right)^{2}\right]+2 \mu_{\mathrm{c}} \cos \beta \Phi_{\mathrm{c}}
\end{aligned}
$$

Here the spin and charge velocities $v_{s, c}$ and the parameters $\beta$ and $\mu_{c}$ are functions of $U$ and $V_{j}$. The Hamiltonian (7) exhibits spin-charge separation: $\mathcal{H}_{\mathrm{c}, \mathrm{s}}$ describe charge and spin degrees of freedom respectively, which are independent of one another. The pure Hubbard model corresponds to the limit $\beta \rightarrow 1$ and the effect of $V_{j}$ is to decrease the value of $\beta$. From the form (7) we can deduce a number of important properties. Firstly, the spin sector is gapless and is described by a free bosonic theory. Hence correlation functions involving (vertex operators of) the spin boson $\Phi_{s}$ and its dual field $\Theta_{s}$ can be calculated by standard methods [8]. Excitations in the spin sector are scattering states of gapless, chargeless spin $\frac{1}{2}$ objects called spinons. The charge sector of (7) is a Sine-Gordon model (SGM). Here excitations in the regime $\beta>\frac{1}{\sqrt{2}}$ are scattering states of gapped charge $\pm e$ excitations called soliton and antisoliton respectively. In the context of the halffilled Mott insulator these are also known as holon and antiholon. Soliton and antisoliton have massive relativistic dispersions,

$$
E(P)=\sqrt{M^{2}+v_{\mathrm{c}}^{2} P^{2}},
$$

where $M$ is the single-particle gap. For $V_{j}=0, j \geq 3$ the gap scales as

$$
M \approx \frac{8 t}{\sqrt{2 \pi}} \sqrt{g(1+x)}\left(\frac{1-x}{1+x}\right)^{(g x+2) / 4 g x},
$$

where we have fixed the constant factor by comparing to the exact result for the Hubbard model, and where

$$
\begin{aligned}
& x=\left[1-\left(\frac{U-2 V_{1}+2 V_{2}}{U+6 V_{2}+2 V_{2}}\right)^{2}\right]^{1 / 2}, \\
& g=\left(U+6 V_{1}+2 V_{2}\right) / 2 \pi t .
\end{aligned}
$$

We note that the gap vanishes on the critical surface $U-2 V_{1}+2 V_{2}=0$ separating the Mott-insulating phase with gapless spin excitations from another phase with a spin gap. 
In the regime $0<\beta<1 / \sqrt{2}$, soliton and antisoliton attract and can form bound states. In the SGM these are usually known as "breathers" and correspond to excitons in the underlying extended Hubbard lattice model. There are

$$
N=\left[\frac{1-\beta^{2}}{\beta^{2}}\right]
$$

different types of excitons, where $[x]$ in (11) denotes the integer part of $x$. The exciton gaps are given by

$$
M_{n}=2 M \sin (n \pi \xi / 2), \quad n=1, \ldots, N,
$$

where

$$
\xi=\frac{\beta^{2}}{1-\beta^{2}}
$$

\section{B. Quarter Filled Band}

In the quarter filled case there are no simple Umklapp processes that can open a gap in the charge sector. As a result the quarter-filled extended Hubbard model is metallic in the weak coupling regime. However, integrating out the high energy degrees or freedom generates "double Umklapp" processes involving four electron creation and annihilation operators each $[9,10]$. For small $U, V_{j}$ these processes are irrelevant, but increasing $U, V_{j}$ decreases their scaling dimension until the double Umklapp term eventually becomes relevant. The low-energy effective Hamiltonian is identical to (7), but the relations between the Fermi operators and the Bose fields are different

$$
\begin{aligned}
& L_{\sigma}^{\dagger}(x)=\frac{\eta_{\sigma}}{\sqrt{2 \pi}} e^{i f_{\sigma} \pi / 4} \exp \left(-\frac{i}{4}\left[\frac{\beta}{2} \Phi_{c}-\frac{2}{\beta} \Theta_{c}\right]\right) \exp \left(-\frac{i f_{\sigma}}{4}\left[\Phi_{s}-\Theta_{s}\right]\right) \\
& R_{\sigma}^{\dagger}(x)=\frac{\eta_{\sigma}}{\sqrt{2 \pi}} e^{i f_{\sigma} \pi / 4} \exp \left(\frac{i}{4}\left[\frac{\beta}{2} \Phi_{c}+\frac{2}{\beta} \Theta_{c}\right]\right) \exp \left(\frac{i f_{\sigma}}{4}\left[\Phi_{s}+\Theta_{s}\right]\right) .
\end{aligned}
$$

Although the low-energy effective Hamiltonian is the same as for the half-filled Mott insulator, the physical properties in the quarter-filled case are rather different. Firstly, the insulating state emerging for sufficiently large $U, V_{j}$ at quarter filling is generated by a different physical mechanism (double Umklapp scattering) as compared to half filling (Umklapp scattering) and concomitantly is referred to as a $4 k_{F}$ charge-density wave insulator in the literature [11]. We adopt this terminology here. Secondly, the quantum numbers of elementary excitations in the charge sector are different. Like for the half-filled case the elementary excitations in the charge sector are a soliton/antisoliton doublet, but now they carry fractional charge $\pm \frac{e}{2}$. A simple way to see this is to recall that the conserved topological charge in the SGM is defined as

$$
Q=\frac{\beta}{2 \pi} \int_{-\infty}^{\infty} d x \partial_{x} \Phi_{c}
$$


The soliton has topological charge -1 and the antisoliton +1 . A simple calculation shows that the right moving fermion creates two solitons

$$
Q R_{\sigma}^{\dagger}(x)|0\rangle=-2 R_{\sigma}^{\dagger}(x)|0\rangle
$$

This implies that fermion number one corresponds to topological charge 2 and hence solitons have fractional charge. The elementary excitations in the spin sector are again a pair of gapless, chargeless spin $\pm \frac{1}{2}$ spinons.

\section{The Method}

Due to spin charge separation a general local operator $\mathcal{O}(t, x)$ can be represented as a product of a charge and a spin piece $\mathcal{O}=\mathcal{O}_{c} \mathcal{O}_{s}$. As a result correlation functions factorize as well

$$
\left\langle 0\left|\mathcal{O}^{\dagger}(t, x) \mathcal{O}(0)\right| 0\right\rangle={ }_{c}\left\langle 0\left|\mathcal{O}_{c}^{\dagger}(t, x) \mathcal{O}_{c}(0)\right| 0\right\rangle_{c s}\left\langle 0\left|\mathcal{O}_{s}^{\dagger}(t, x) \mathcal{O}_{s}(0)\right| 0\right\rangle_{s},
$$

where $|0\rangle_{s, c}$ are the vacua in the spin and charge sectors respectively. The correlation function in the spin sector is easily evaluated as we are dealing with a free theory.

\section{Correlation Functions in the Charge Sector}

Let us now discuss how to calculate two point functions in the SGM describing the charge sector of the theory. One first constructs a spectral representation and then utilizes integrability to determine the matrix elements of the operator under consideration between the ground state and excited states. This method is known as the "Form Factor Bootstrap Approach" [12,13]. In order to utilize the spectral representation we need to specify a basis of eigenstates of the Hamiltonian ( 7 ). Such a basis is given by scattering states of solitons, antisolitons and breathers. In order to distinguish these we introduce labels $B_{1}, B_{2}, \ldots B_{N}, s, \bar{s}$. As usual for particles with relativistic dispersion, it is useful to introduce a rapidity variable $\theta$ to parametrize energy and momentum

$$
\begin{aligned}
E_{s}(\theta) & =M \cosh \theta, P_{s}(\theta)=\left(M / v_{\mathrm{c}}\right) \sinh \theta, \\
E_{\bar{s}}(\theta) & =M \cosh \theta, P_{\bar{s}}(\theta)=\left(M / v_{\mathrm{c}}\right) \sinh \theta, \\
E_{B_{n}}(\theta) & =M_{n} \cosh \theta, P_{B_{n}}(\theta)=\left(M_{n} / v_{\mathrm{c}}\right) \sinh \theta,
\end{aligned}
$$

where the breather gaps $M_{n}$ are given by (12). A basis of scattering states of solitons, antisolitons and breathers can be constructed by means of the Zamolodchikov-Faddeev (ZF) algebra, which is the extension of the algebra of creation and annihilation operators for free fermions or bosons to the case of interacting particles with factorizable scattering. The ZF algebra is based on the knowledge of the exact spectrum and scattering matrix of the model [14]: For the SGM the 
ZF operators (and their hermitian conjugates) satisfy the following "generalized commutation relations"

$$
\begin{aligned}
& Z^{\epsilon_{1}}\left(\theta_{1}\right) Z^{\epsilon_{2}}\left(\theta_{2}\right)=S_{\epsilon_{1}^{\prime}, \epsilon_{2}^{\prime}}^{\epsilon_{1}, \epsilon_{2}}\left(\theta_{1}-\theta_{2}\right) Z^{\epsilon_{2}^{\prime}}\left(\theta_{2}\right) Z^{\epsilon_{1}^{\prime}}\left(\theta_{1}\right) \\
& Z_{\epsilon_{1}}^{\dagger}\left(\theta_{1}\right) Z_{\epsilon_{2}}^{\dagger}\left(\theta_{2}\right)=Z_{\epsilon_{2}^{\prime}}^{\dagger}\left(\theta_{2}\right) Z_{\epsilon_{1}^{\prime}}^{\dagger}\left(\theta_{1}\right) S_{\epsilon_{1}, \epsilon_{2}}^{\epsilon_{1}^{\prime}, \epsilon_{2}^{\prime}}\left(\theta_{1}-\theta_{2}\right) \\
& Z^{\epsilon_{1}}\left(\theta_{1}\right) Z_{\epsilon_{2}}^{\dagger}\left(\theta_{2}\right)=Z_{\epsilon_{2}^{\prime}}^{\dagger}\left(\theta_{2}\right) S_{\epsilon_{2}, \epsilon_{1}^{\prime}}^{\epsilon_{2}^{\prime}, \epsilon_{1}}\left(\theta_{2}-\theta_{1}\right) Z^{\epsilon_{1}^{\prime}}\left(\theta_{1}\right)+(2 \pi) \delta_{\epsilon_{2}}^{\epsilon_{1}} \delta\left(\theta_{1}-\theta_{2}\right)
\end{aligned}
$$

Here $S_{\epsilon_{1}^{\prime}, \epsilon_{2}^{\prime}}^{\epsilon_{1}, \epsilon_{2}}(\theta)$ are the known (factorizable) two-particle scattering matrices [14] and $\varepsilon_{j}=s, \bar{s}, B_{1}, \ldots, B_{[1 / \xi]}$. Using the ZF operators a Fock space of states can be constructed as follows. The vacuum is defined by

$$
Z_{\varepsilon_{i}}(\theta)|0\rangle=0 .
$$

Multiparticle states are then obtained by acting with strings of creation operators $Z_{\epsilon}^{\dagger}(\theta)$ on the vacuum

$$
\left|\theta_{n} \ldots \theta_{1}\right\rangle_{\epsilon_{n} \ldots \epsilon_{1}}=Z_{\epsilon_{n}}^{\dagger}\left(\theta_{n}\right) \ldots Z_{\epsilon_{1}}^{\dagger}\left(\theta_{1}\right)|0\rangle
$$

In this basis the resolution of the identity is given by

$$
\mathbb{1}=|0\rangle\left\langle 0\left|+\sum_{n=1}^{\infty} \sum_{\epsilon_{i}} \int_{-\infty}^{\infty} \frac{d \theta_{1} \ldots d \theta_{n}}{(2 \pi)^{n} n !}\right| \theta_{n} \ldots \theta_{1}\right\rangle_{\epsilon_{n} \ldots \epsilon_{1}}{ }^{\epsilon_{1} \ldots \epsilon_{n}}\left\langle\theta_{1} \ldots \theta_{n}\right|,
$$

and the following spectral representation of the two-point function of the operator $\mathcal{O}$ holds

$$
\begin{aligned}
& \left\langle\mathcal{O}(x, t) \mathcal{O}^{\dagger}(0,0)\right\rangle= \\
& \sum_{n=1}^{\infty} \sum_{\epsilon_{i}} \int \frac{d \theta_{1} \ldots d \theta_{n}}{(2 \pi)^{n} n !} \exp \left(i \sum_{j=1}^{n} P_{j} x-E_{j} t\right)\left|\left\langle 0|\mathcal{O}(0,0)| \theta_{n} \ldots \theta_{1}\right\rangle_{\epsilon_{n} \ldots \epsilon_{1}}\right|^{2} .
\end{aligned}
$$

Here $P_{j}$ and $E_{j}$ are given by

$$
P_{j}=\frac{M_{\epsilon_{j}}}{v_{\mathrm{c}}} \sinh \theta_{j}, E_{j}=M_{\epsilon_{j}} \cosh \theta_{j},
$$

and the formfactors of the operator $\mathcal{O}$ are denoted by

$$
f^{\mathcal{O}}\left(\theta_{1} \ldots \theta_{n}\right)_{\epsilon_{1} \ldots \epsilon_{n}} \equiv\left\langle 0|\mathcal{O}(0,0)| \theta_{n} \ldots \theta_{1}\right\rangle_{\epsilon_{n} \ldots \epsilon_{1}} .
$$

The form factors can be calculated by solving a Riemann-Hilbert problem involving the exact scattering matrix of the SGM and are known for many operators. 
Our conventions in (28) are such that $M_{s}=M_{\bar{s}}=M$ and $M_{B_{n}}=M_{n}$. We are mainly interested in the Fourier transforms of the retarded space and time dependent correlation functions. These take the following general form

$$
\begin{aligned}
\chi^{\mathcal{O}}(\omega, q)=-2 \pi & \sum_{n=1}^{\infty} \sum_{\epsilon_{2}} \int \frac{d \theta_{1} \ldots d \theta_{n}}{(2 \pi)^{n} n !}\left|f^{\mathcal{O}}\left(\theta_{1} \ldots \theta_{n}\right)_{\epsilon_{1} \ldots \epsilon_{n}}\right|^{2} \\
& {\left[\frac{\delta\left(q-\sum_{j} M_{\epsilon_{j}} \sinh \theta_{j} / v_{\mathrm{c}}\right)}{\omega-\sum_{j} M_{\epsilon_{j}} \cosh \theta_{j}+i \eta}-\frac{\delta\left(q+\sum_{j} M_{\epsilon_{j}} \sinh \theta_{j} / v_{\mathrm{c}}\right)}{\omega+\sum_{j} M_{\epsilon_{j}} \cosh \theta_{j}+i \eta}\right] . }
\end{aligned}
$$

\section{Optical Conductivity}

The optical conductivity was calculated in Refs $[17,18,19,21]$. In the field-theory limit, the electrical current operator is given by

$$
\mathcal{J}(t, x)=\sqrt{A^{\prime}} \partial_{t} \phi_{\mathrm{c}}
$$

where $A^{\prime}>0$ is a non-universal constant. The expression for the current operator is the same for the half-filled and the quarter filled bands. As seen from Eq. (31), the current operator does not couple to the spin sector. This shows that spinons do not contribute to the optical conductivity in the field theory limit. Hence, the calculation of the optical conductivity has been reduced to the evaluation of the retarded current-current correlation function in the charge sector. The real part of the optical conductivity $(\omega>0)$ has the following spectral representation

$$
\begin{gathered}
\sigma(\omega)=\frac{2 \pi^{2}}{L a_{0} \omega} \sum_{n=1}^{\infty} \sum_{\epsilon_{i}} \int \frac{d \theta_{1} \ldots d \theta_{n}}{(2 \pi)^{n} n !}\left|f^{\mathcal{J}}\left(\theta_{1} \ldots \theta_{n}\right)_{\epsilon_{1} \ldots \epsilon_{n}}\right|^{2} \\
\delta\left(\sum_{k} \frac{M_{\epsilon_{k}}}{v_{\mathrm{c}}} \sinh \theta_{k}\right) \delta\left(\omega-\sum_{k} M_{\epsilon_{k}} \cosh \theta_{k}\right) .
\end{gathered}
$$

In Refs. $[12,13]$ integral representations for the form factors of the current operator in the SGM were derived. Using these results we can determine the first few terms of the expansion (32). From (32) it is easy to see for any given frequency $\omega$ only a finite number of intermediate states will contribute: the delta function forces the sum of single-particle gaps $\sum_{j} M_{\epsilon_{j}}$ to be less than $\omega$. Expansions of the form (32) are usually found to exhibit a rapid convergence, which can be understood in terms of phase space arguments $[15,16]$. Therefore we expect that summing the first few terms in (32) will give us good results over a large frequency range.

Using the transformation property of the current operator under charge conjugation one finds that many of the form factors in (30) actually vanish. In particular, only the "odd" breathers $B_{1}, B_{3}, \ldots$ (assuming they exist, i.e., $\beta$ is sufficiently small) couple to the current operator. The first few non-vanishing terms of the 
spectral representation (32) are

$$
\frac{\sigma(\omega)}{A}=\sum_{n=1}^{[(N+1) / 2]} \sigma_{B_{2 n-1}}(\omega)+\sigma_{s \bar{s}}(\omega)+\sigma_{B_{1} B_{2}}(\omega)+\ldots
$$

Here $A=A^{\prime} v_{\mathrm{c}} / L a_{0}$ and $\sigma_{B_{n}}(\omega), \sigma_{s \bar{s}}(\omega)$ and $\sigma_{B_{1} B_{2}}(\omega)$ are the contributions of the odd breathers, the soliton-antisoliton continuum and the $B_{1} B_{2}$ breather-breather continuum respectively. The latter of course exists only if $N \geq 2$. We find

$$
\begin{gathered}
\sigma_{B_{2 n-1}}(\omega)=\frac{\pi}{M_{2 n-1}^{2}} f_{2 n-1} \delta\left(\omega-M_{2 n-1}\right) \\
f_{m}=4 M^{2} \xi^{2} \sin (m \pi \xi) \prod_{n=1}^{m-1} \tan ^{2}(\pi n \xi / 2) \\
\exp \left(-2 \int_{0}^{\infty} \frac{d t}{t} \frac{\sinh (t(1-\xi) / 2)}{\sinh (t \xi / 2) \cosh (t / 2)} \frac{\sinh ^{2}(m t \xi / 2)}{\sinh t}\right) .
\end{gathered}
$$

The soliton-antisoliton contribution is [18]

$$
\begin{aligned}
\sigma_{s \bar{s}}(\omega)=\frac{4 \sqrt{\omega^{2}-4 M^{2}} \Theta(\omega-2 M)}{\omega^{2}[\cos (\pi / \xi)+\cosh (\theta / \xi)]} \\
\quad \exp \left(\int_{0}^{\infty} \frac{d t}{t} \frac{\sinh [t(1-\xi) / 2][1-\cos (t \theta / \pi) \cosh t]}{\sinh (t \xi / 2) \cosh (t / 2) \sinh t}\right)
\end{aligned}
$$

where $\theta=2 \operatorname{arccosh}(\omega / 2 M)$. The result for the $B_{1} B_{2}$ breather-breather continuum is given in Ref. [21]. As a function of the parameter $\beta$, the optical conductivity behaves as follows.

- $1 \geq \beta^{2}>1 / 2$ :

In this regime the optical spectrum consists of a single "band" corresponding to (multi) soliton-antisoliton states above a threshold $\Delta=2 M$. The absorption band increases smoothly above the threshold $\Delta$ in a universal square root fashion

$$
\sigma(\omega) \propto \sqrt{\omega-\Delta} \text { for } \omega \rightarrow \Delta^{+}
$$

In Fig. 1 we plot the leading contributions for the case $\beta^{2}=0.9$. Clearly, the four-particle contribution is negligible at low frequencies.

- $1 / 2 \geq \beta^{2}>1 / 3$ :

Here the optical spectrum contains one band and one excitonic breather peak below the optical gap $\Delta=2 M$ at the energy $\omega_{B_{1}}=M_{1}$. The optical weight is progressively transferred from the band to the breather as $\beta^{2}$ decreases down to $1 / 3$. The absorption band again increases in a square-root fashion 


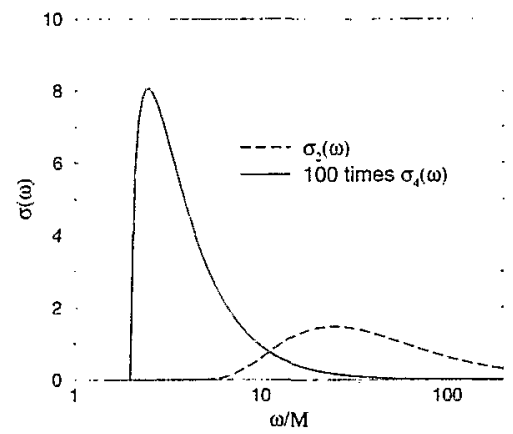

Figure 1: Optical conductivity for $\beta^{2}=0.9$. Shown are the dominant contributions at low frequencies: the soliton-antisoliton part $\sigma_{2}(\omega)$ and the two soliton - two antisoliton contribution $\sigma_{4}(\omega)$.

(37) above the threshold for all values of $\beta^{2}$ except $\beta^{2}=1 / 2$, where the breather peak merges with the band. In this case $\sigma(\omega)$ shows a square-root divergence at the absorption threshold

$$
\sigma(\omega) \propto \frac{1}{\sqrt{\omega-\Delta}} \text { for } \omega \rightarrow \Delta^{+}\left(\beta^{2}=1 / 2\right) .
$$

The field theory results discussed here have been compared to Dynamical Density Matrix Renormalization Group (DDMRG) (see e.g. Ref.[20] and references therein) computations of $\sigma(\omega)$ for extended Hubbard models in Refs [17, 21, 22] and good agreement has been found in the appropriate regime of parameters. We note that the DDMRG method can also deal with parameter regimes in the underlying lattice model, to which field theory does not apply. Let us discuss the above results from the point of view of an application to optical conductivity measurements in the Bechgaard salts [23]. There it is found that up to $99 \%$ of the total spectral weight is concentrated in a finite-frequency feature, which has been attributed to Mott physics of the type discussed here [23]. A comparison of (33) to the experimental data gives satisfactory agreement at high frequencies, but the detailed peak structure at low frequencies is not reproduced [18]. A likely source for these differences is the interchain tunneling.

\section{Spectral Function}

\section{A. Half-Filled Mott Insulator}

The zero-temperature spectral function of the half-filled Mott insulator has been studied in many previous works. There have been extensive numerical studies on 
finite size $t-J$ and Hubbard models e.g. Refs $[24,5]$. The limit where the singleparticle gap is much larger than the bandwidth was treated in Refs [25]. This regime is complementary to the case we address here. The weak-coupling limit we are interested in was studied in Refs [26, 27, 28], where a conjecture for the spectral function was put forward. Here we derive these results by means of an exact, systematic method. In what follows we will for simplicity fix $\beta=1$, i.e. deal with the Hubbard model only.

\section{Zero Temperature}

The single particle Green's function is calculated by following the steps outlined above [29]. The creation and annihilation operators for right and left moving fermions factorize into spin and charge pieces upon bosonization (3). The spin part is easily calculated: In imaginary time we have

$$
\left\langle\exp \left(-\frac{i f_{\sigma}}{4}\left[\Phi_{s}+\Theta_{s}\right]\right) \exp \left(\frac{i f_{\sigma}}{4}\left[\Phi_{s}+\Theta_{s}\right]\right)\right\rangle=\frac{1}{\sqrt{v_{s} \tau-i x}} .
$$

The correlation function in the charge sector is calculated by means of the form factor bootstrap approach. Taking into account only processes involving one scliton. we obtain

$$
\begin{aligned}
&\left\langle\exp \left(-\frac{i}{4}\left[\Phi_{c}+\Theta_{c}\right]\right) \exp \left(\frac{i}{4}\left[\Phi_{c}+\Theta_{c}\right]\right)\right\rangle \simeq \\
& \frac{Z_{0}}{\sqrt{v_{c} \tau-i x}} \exp \left(-\frac{M}{v_{c}} \sqrt{x^{2}+v_{c}^{2} \tau^{2}}\right),
\end{aligned}
$$

where $Z_{0} \approx 0.9218$ [30]. The corrections to (40) involve intermediate states with three particles and are negligible at long distances/low energies. In Fourier space we obtain after analytical continuation to real frequencies

$$
\begin{aligned}
& \quad G^{(R)}\left(\omega, k_{F}+q\right) \simeq \\
& -Z_{0} \frac{\omega+v_{c} q}{\sqrt{M^{2}+v_{c}^{2} q^{2}-\omega^{2}}}\left[\left(M+\sqrt{M^{2}+v_{c}^{2} q^{2}-\omega^{2}}\right)^{2}-\frac{v_{c}-v_{s}}{v_{c}+v_{s}}\left(\omega+v_{c} q\right)^{2}\right]^{-\frac{1}{2}} .
\end{aligned}
$$

We note that the charge velocity $v_{c}$ is larger than the spin velocity $v_{s}$. The spectral function is obtained from the imaginary part of the single particle Green's function (41). In the case $v_{s}=v_{c}=v$ it takes the simple form

$$
A_{R}\left(\omega, k_{F}+q\right)=-\frac{1}{\pi} \operatorname{Im} G^{(R)}\left(\omega, k_{F}+q\right)=\frac{Z_{0} m}{\pi|\omega-v q|} \frac{\Theta\left(|\omega|-\sqrt{m^{2}+v^{2} q^{2}}\right)}{\sqrt{\omega^{2}-m^{2}-v^{2} q^{2}}} .
$$

In Fig. 2 we plot the spectral function in the case $v_{s}=0.8 v_{c}$ and $\beta=1$ both in a density plot and in a series of constant $q$ scans. There is a continuum of 

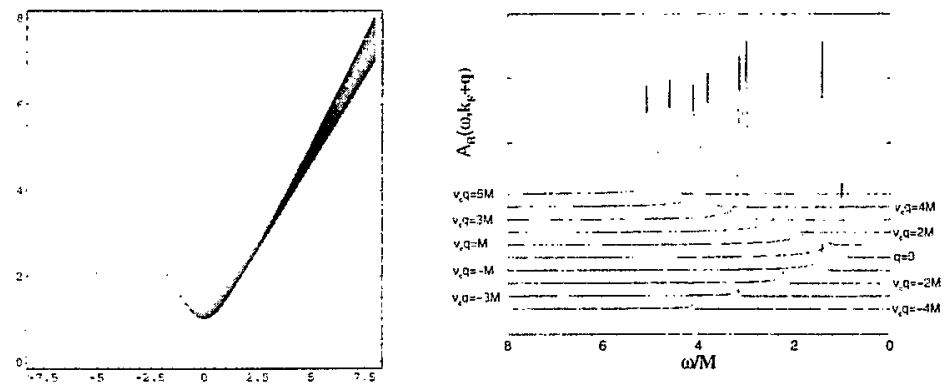

Figure 2: Spectral function for a half-filled Mott insulator with $v_{s}=0.8 v_{c}$ and $\beta=1$.

states above the Mott gap, which is smallest at $k_{F}$. The most striking feature is the presence of two distinct "peaks" dispersing with velocities $v_{s}$ and $v_{c}$ respectively. Most of the spectral weight is concentrated in these features, which are a direct manifestation of Spin-Charge Separation. The lower (higher) energy feature corresponds to the situation where all the momentum is carried by the spin (charge) sector. Concomitantly the high/low energy feature is referred to as antiholon/spinon peak. Neither peak is sharp but has intrinsic width (more precisely, they correspond to square root divergences).

\section{Finite Temperature $T \ll M$}

It is possible to extend the results for the spectral function to small temperatures $T \lesssim M[31]$. The method is a straightforward generalization of the one we employed for $T=0$. As $T \lesssim M$ the effects of temperature on the charge piece of the correlation function are small, but the spin piece can be affected quite strongly because the spinons are gapless. The spectral function in the vicinity of $k_{F}$ can be represented as

$$
\begin{aligned}
& A_{R}\left(\omega, k_{F}+q\right) \approx \\
& \mathcal{A} \int_{-\infty}^{\infty} d z e^{z / 2}\left[\tilde{g}_{s}(\omega-c(z), q-s(z))+e^{-c(z) / T} \tilde{g}_{s}(\omega+c(z), q+s(z))+\left\{\begin{array}{c}
\omega \rightarrow-\omega \\
q \rightarrow-q
\end{array}\right\}\right],
\end{aligned}
$$

where $\mathcal{A}=\sqrt{\frac{\pi \Delta}{v_{s}}} \frac{Z_{2}}{(2 \pi)^{3}}, c(z)=\Delta \cosh z, s(z)=\left(\Delta / v_{s}\right) \sinh z$ and

$$
\tilde{g}_{s}(\omega, q)=\frac{\left(v_{s} / \pi T\right)^{3 / 2}}{v_{s} \sqrt{2}} \operatorname{Re}\left[\sqrt{-2 i} B\left(\frac{1}{4}-i \frac{\omega+v_{s} q}{4 \pi T}, \frac{1}{2}\right)\right] \delta\left(\omega-v_{s} q\right) .
$$



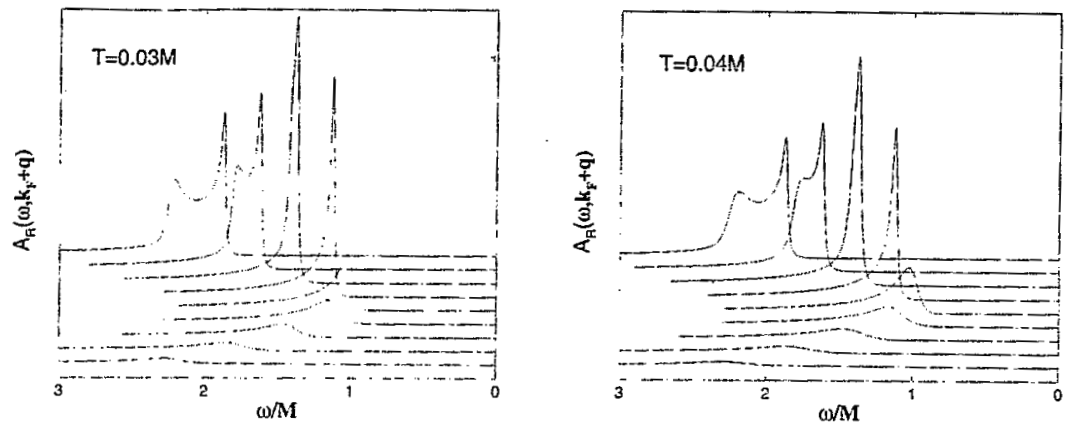

Figure 3: Spectral function for a half-filled Mott insulator with $v_{s}=0.5 v_{c}$ and $\beta=1$ a'temperatures $T=0.03 M$ and $T=0.04$ respectively.

In Fig. 3 we plot the spectral function for $v_{s}=0.5 v_{c}$ and $\beta=1$ at temperatures of 0.03 and 0.04 times the single particle gap. A significant temperature dependence of the "charge" peak is apparent. It gets damped rather strongly at temperatures that are still small compared to the gap. 'This may make an unambiguous detection of SC-separation by ARPES more difficult as the experiments are done at elevated temperatures (room temperature for $\mathrm{Sr}_{2} \mathrm{CuO}_{3}$ ) in order to avoid charging effects.

\section{B. Quarter-Filled CDW Insulator}

The single-particle Green's function in the quarter-filled case can be determined by the same method [32]. The spin sector is again gapless and the spin part of the Green's function is easily determined. The charge piece is again analyzed by means of the form factor bootstrap approach. The difference to the half-filled case is that the charge piece of the single-electron operator (14) now couples to at least two (anti)solitons. Neglecting contributions of four or more particles in the charge sector we obtain the following result for the single-particle Green's function in the vicinity of $k_{F}$

$$
\begin{aligned}
& G^{(R)}\left(\omega, k_{F}+q\right)=-Z \sqrt{\frac{2 v_{c}^{2}}{v_{c}+v_{s}}} \int_{-\infty}^{\infty} \frac{d \theta}{\sqrt{2 \pi}} \frac{|G(2 \theta)|^{2}}{\sqrt{c(\theta)}} \frac{\omega+v_{c} q}{\sqrt{c^{2}(\theta)-s^{2}}} \\
& {\left[\left(c(\theta)+\sqrt{c^{2}(\theta)-s^{2}}\right)^{2}-\frac{\left(\omega+v_{c} q\right)^{2}}{\alpha}\right]^{-\frac{1}{2}} }
\end{aligned}
$$

where $s^{2}=\omega^{2}-v_{c}^{2} q^{2}, c(\theta)=2 M \cosh \theta, \alpha=\left(v_{c}+v_{s}\right) /\left(v_{c}-v_{s}\right)$. In Fig. 4 we plot the spectral function $A_{R}\left(\omega, k_{F}+q\right)$ as a function of $\omega$ for $v_{s}=0.8 v_{c}$ and different values of $q$. Clearly the spectral function is rather featureless and there are no singularities. Furthermore, in contrast to the half-filled Mott insulator discussed 
above, thcre are no dispersing features associated separately with $v_{c}$ and $v_{s}$. The absence of any distinct features is clearly related to the fact that the electron has "fallen apart" into at least three pieces.

Just above the threshold at $s^{2}=\omega^{2}-v^{2} q^{2}=4 M^{2}$ one can approximate $|G(2 \theta)|=\left|C_{1} \sinh \theta\right|$, so that for $v_{c}=v_{s}$

$$
A_{R}(\omega, q) \approx Z \sqrt{\frac{v}{4 \pi M}} \frac{1}{|\omega-v q|}\left(\frac{s}{2 M}-1\right) .
$$

Thus the spectral weight increases linearly with $s-2 M$ above the threshold. The

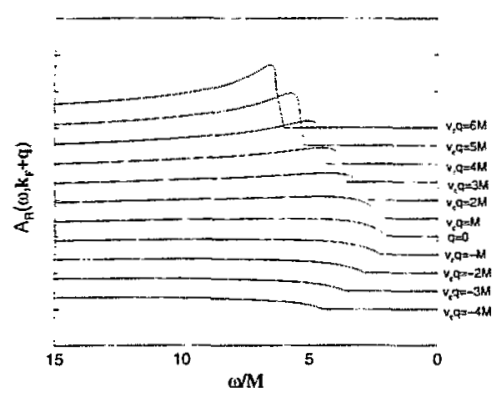

Figure 4: $A_{R}\left(\omega, k_{F}+q\right)$ as a function of $\omega / M$ for $v_{s}=0.8 v_{c}$. The curves for different $q$ are offset.

tunneling density of states for $v_{c}=v_{s}$ is

$$
\rho(\omega)=\frac{2 Z}{\sqrt{v M}} \int_{0}^{\operatorname{arccosh}(\omega / 2 M)} \frac{d \theta}{\sqrt{2 \pi}} \frac{|G(2 \theta)|^{2}}{\sqrt{2 \cosh \theta}},
$$

and displays a roughly linear increase after an initial $(\omega-2 M)^{3 / 2}$ behaviour just above the threshold at $\omega=2 M$.

Let us now turn to a comparison with experiments. $\mathrm{PrBa}_{2} \mathrm{Cu}_{3} \mathrm{O}_{7}$ (P123) is a quarter-filled quasi-1D cuprate, to which the theory presented here may have some relevance. The ARPES data for P123 (Fig. 3 of [6]) show a single, very broad, dispersing feature that is asymmetric around $k_{F}$. If we interpret the underlying increase in intensity in the data as background, the signal has a form similar to Fig. 4. In order to assess whether the theory presented here is indeed relevant to P123, it would be interesting to extract a value $\Delta_{\mathrm{PE}}^{\left(\frac{1}{4}\right)}$ for the gap from the ARPES data and compare it to gaps seen in optical measurements $\Delta_{\text {opt }}^{\left(\frac{1}{4}\right)}$ and the thermal activation gap $\Delta_{\mathrm{T}}^{\left(\frac{1}{4}\right)}$ extracted e.g. from the dc conductivity. The theory presented here predicts

$$
\Delta_{\mathrm{opt}}^{\left(\frac{1}{4}\right)}=2 \Delta_{\mathrm{T}}^{\left(\frac{1}{4}\right)}=\Delta_{\mathrm{PE}}^{\left(\frac{1}{4}\right)} .
$$


This is in contrast to the case of the half-filled Mott insulator, where one has

$$
\Delta_{\mathrm{opt}}^{\left(\frac{1}{2}\right)}=2 \Delta_{\mathrm{T}}^{\left(\frac{1}{2}\right)}=2 \Delta_{\mathrm{PE}}^{\left(\frac{1}{2}\right)}
$$

\section{Luttinger's Theorem}

In all cases we have discussed, the Green's functions have branch cuts but no poles.

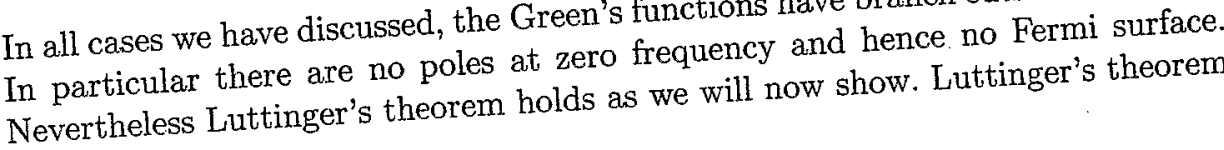
reads [33]

$$
\frac{N}{V}=\int_{G(0, k)>0} \frac{d^{D} k}{(2 \pi)^{D}}
$$

where the integration is over the interior of the region defined by either singularities or zeroes of the single-particle Green's function. The former is the case for a Fermi liquid whereas the latter is the case at hand. The equality (49) is intera Fermi liquid whereas the latter is the case at hand. The equale is independent
esting, because it implies that the integral on the right hand side in the volume of
of electron-electron interactions. In a Fermi liquid this means that the the Fermi surface is unaffected by interactions. The Green's functions we have discussed above all fulfill (49) by virtue of them having zeroes at the position of the non-interacting Fermi surface.

This property follows from (i) the fact that the spin sector is gapless, which implies that $\left\langle R_{\sigma}(\tau, x) L_{\sigma}^{\dagger}(0)\right\rangle=0$ and (ii) Lorentz invariance of the low-energy effective theory, which implies that

$$
\left\langle\Psi_{\sigma}(\tau, x) \Psi_{\sigma}^{\dagger}(0)\right\rangle \sim \exp ( \pm i \phi) \mathcal{R}(r) ; \Psi=R, L
$$

Here $r$ and $\phi$ are polar coordinates and $\mathcal{R}$ denotes the radial part of the correlation function. As we are dealing with an insulating state we have $\mathcal{R}(r) \propto \exp (-\Delta r)$ at large distances and hence $\int d r \mathcal{R}(r) r$ is finite. Thus

$$
G_{R, L}(0,0)=\int_{-\pi}^{\pi} d \phi \exp ( \pm i \phi) \int d r \mathcal{R}(r) r=0 .
$$

For a metallic state the $r$ integral would diverge and the Green's function would have a singularity rather than a zero.

\section{Interchain Tunneling}

Let us now consider a quasi one dimensional situation of Hubbard chains weakly coupled by a long-ranged interchain tunneling

$$
\begin{aligned}
H & =\sum_{l} H^{(l)}+\sum_{l, m \neq l . n, \sigma} t_{\perp}^{l m} c_{n . \sigma}^{(l) \dagger} c_{n . \sigma}^{(m)}+\text { h.c. } \\
H^{(l)} & =-t \sum_{n, \sigma} c_{n . \sigma}^{(l) \dagger} c_{n+1 . \sigma}^{(l)}+\text { h.c. }+U \sum_{n} n_{j . \uparrow}^{(l)} n_{j . \downarrow}^{(l)} .
\end{aligned}
$$




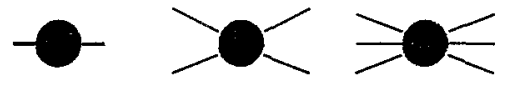

Figure 5: Elements of the diagrammatic perturbation theory in the interchain tunneling.

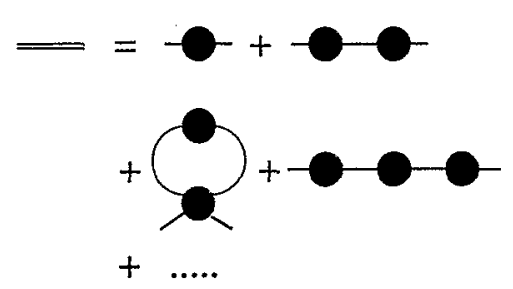

Figure 6: Diagrammatic expansion for the single-particle Green's function of coupled chains.

We allow the interchain tunneling to be long-ranged in order to be able to carry out a controlled expansion in the case where the Fourier transform $\tilde{t}_{\perp}(\vec{k})$ of the interchain tunneling becomes of the same order as the 1D Mott gap $M$ (see the discussion below). For simplicity we take $t_{\perp}$ long-ranged only in the direction perpendicular to the chains, although it is straightforward to generalize all formulas to the an interchain tunneling of the form.

$$
\sum_{l, m \neq l, n, p, \sigma}\left[t_{\perp}\right]_{n p}^{l m} c_{n, \sigma}^{(l) \dagger} c_{p, \sigma}^{(m)}+\text { h.c. . }
$$

\section{VII.1 A. Expansion around uncoupled chains}

Following the analogous treatment for the case of coupled Luttinger liquids $[34,35]$ we take the interchain tunneling into account in a perturbative expansion. The building blocks of this expansion are the $n$-point functions of fermion operators for uncoupled chains, which are represented pictorially in Fig. 5. The full singleparticle Green's function is given in terms of a diagrammatic expansion, the first few terms of which are shown in Fig. 6

Unlike for Luttinger liquids it is extremely difficult to determine four-point functions for $1 \mathrm{D}$ Mott insulators. On the other hand, it is trivial to sum all diagrams involving only two-point functions of uncoupled chains. This approximation is known as RPA and goes back a long way [36]. The small parameter making RPA a controlled approximation for any form of the interchain tunneling is the ratio of the interchain tunneling to either the temperature or the Mott gap. Within RPA 


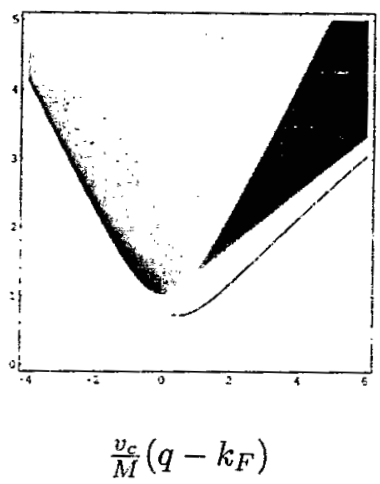

Figure 7: Spectral Function for fixed $\vec{k}$.

the single-particle Green's function is given by

$$
\begin{aligned}
G_{3 D}(\omega, q, \vec{k}) & =\frac{G_{1 D}(\omega, q)}{1-t_{\perp}(\vec{k}) G_{1 D}(\omega, q)} \\
t_{\perp}(\vec{k}) & =\sum_{m} t_{\perp}^{l m} \exp \left(i \vec{k} \cdot\left[\vec{R}_{l}-\vec{R}_{m}\right]\right) .
\end{aligned}
$$

The RPA Green's function $G_{3 \mathrm{D}}(\omega, q, \vec{k})$ has the interesting property that it has a pole, which corresponds to a bound state of an antiholon and a spinon with the quantum numbers of an electron. For small interchain tunneling this bound state still has a gap. Fig. 7 is a density plot of $G_{3 D}(\omega, q, \vec{k})$ as a function of $\omega$ and $q$ for a fixed value of $\vec{k}$ and hence a fixed value of $t_{\perp}(\vec{k})$. At energies above the Mott gap there is a continuum of states, which is similar in nature to the result for uncoupled chains. In the Mott gap the coherent electronic mode is visible.

\section{B. Formation of a Fermi surface}

As long as the "binding energy" of the electronic bound state is small, RPA is a controlled approximation for any form of the interchain tunneling [35]. However, in the most interesting situation when the gap of the bound states becomes very small, RPA becomes uncontrolled; there is no longer any small expansion parameter for a generic $t_{\perp}(\vec{k})$. An exception is the case of a long-ranged interchain tunneling: here the support of $t_{\perp}(\vec{k})$ becomes very small, so that any integration over the transverse momentum generates a small volume factor proportional to the inverse range of the hopping. Recalling that RPA takes into account all terms not involving any integrations over the transverse hopping (i.e. "loops"), we conclude that RPA is the leading term in a controlled loop expansion.

Increasing $t_{\perp}(\vec{k})$ in RPA reduces the gap of the electronic bound state, until it eventually vanishes and electron and hole pockets are formed: we have crossed 


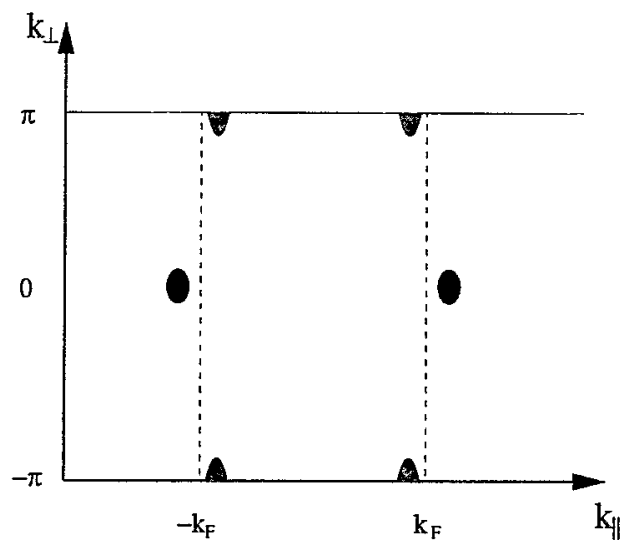

Figure 8: Fermi surface predicted by the RPA for a 2D square lattice with nearestneighbour interchain tunneling.

over from weakly coupled 1D Mott insulators to an anisotropic Fermi liquid. As an example, let us consider a 2D square lattice with interchain tunneling between nearest neighbour chain only. Here RPA is an uncontrolled approximation, but we still find it instructive to discuss its predictions.

In this case electron pockets are formed in the vicinity of the points $\left( \pm k_{F}, 0\right)$ and hole pockets form around $\left( \pm k_{F}, \pm \pi\right)$. In the electron-hole symmetric case the volume of the electron pockets is precisely the same as the volume of the hole pockets.

We note that the "Chain-DMFT" approach (which approximates the coupled chains system by a single chain in a self-consistent bath) gives an open Fermi surface [37].

\section{Conclusions}

We have discussed the dynamical response of two strongly correlated one dimensional insulators: the half-filled Mott insulator and the quarter-filled $4 k_{F}-\mathrm{CDW}$ insulator. In the low-energy limit both of them exhibit spin-charge separation and it is possible to determine the dynamical response by means of exact methods. The optical conductivity is found to be insensitive to the spin degrees of freedom and also does not differentiate between the half-filled Mott and the quarter-filled $4 k_{F}$ CDW insulator. On the other hand the single-particle Green's function (as well as the dynamical density-density reponse function [38]) probe the physics of both spin and charge sectors and are quite different in the two cases. We also have investigated the effects of interchain tunneling $t_{\perp}$ in a system of infinitely many Mott-insulating chains. We have shown that any $t_{\perp} \neq 0$ leads to the formation of a 
gapped electronic bound state below the Mott gap. Increasing $t_{\perp}$ reduces the gap of this bound state until eventually a Fermi surface in the form of closed electron and hole pockets is generated.

\section{Acknowledgments}

We thank F. Gebhard, E. Jeckelmann, P. Johnson and T. Valla for important discussions. This work was supported by the Department of Energy, Division of Material Science under contract number DE-AC02-98 CH10886.

\section{References}

[1] N. F. Mott, Metal-Insulator Transitions, $2^{\text {nd }}$ ed., Taylor and Francis, London (1990); F. Gebhard, The Mott Metal-Insulator Transition, Springer, Berlin (1997).

[2] N. F. Mott, Proc. Roy. Soc. A 62, 416 (1949); Canad. Jo. Phys. 34, 1356 (1964); Phil. Mag. 6, 287 (1961).

[3] A. Georges, G. Kotliar, W. Krauth and M.J. Rozenberg, Rev. Mod. Phys. 68, $13(1996)$.

[4] C. Bourbonnais and D. Jerome, in "Advances in Synthetic Metals, Twenty years of Progress in Science and Technology", ed. by P. Bernier, S. Lefrant and G. Bidan (Elsevier, New York, 1999), p. 206-301 and references therein. See also cond-mat - 9903101.

[5] C. Kim, A.Y. Matsuura, Z.X. Shen, N. Montoyama, H. Eisaki, S. Uchida, T. Tohyama and S. Maekawa, Phys. Rev. Lett. 77, 4054(1996); H. Fujisawa et al, Phys. Rev. B 59, 7358 (1999); K. Kobayashi et. al., Phys. Rev. Lett. 82, 803 (1999);

[6] T. Mizokawa et. al., Phys. Rev. Lett. 85, 4779 (2000).

[7] S. Biermann, A. Georges, T. Giamarchi and A. Lichtenstein, Proceedings of the NATO ASI "Field Theory of Strongly Correlated Fermions and Bosons in Low - Dimensional Disordered Systems", Windsor, August, 2001.

[8] Bosonization in Strongly Correlated Systems A. O. Gogolin, A. A. Nersesyan and A. M. Tsvelik, Cambridge University Press (1999).

[9] T. Giamarchi, Physica B 230-232, 975 (1997).

[10] H. Yoshioka, M. Tsuchizu and Y. Suzumura, J. Phys. Soc. Jpn 70, 762 (2001).

[11] M. Nakamura, Phys. Rev. B 61, 16377 (2000). 
[12] F.A. Smirnov, Form Factors in Completely Integrable Models of Quantum Field Theory (World Scientific, Singapore, 1992).

[13] M. Karowski and P. Weisz, Nucl. Phys. B139, 455 (1978); H. Babujian, A. Fring, M. Karowski and A. Zapletal, Nucl. Phys. B538, 535 (1999); A. Fring, G. Mussardo and P. Simonetti, Nucl. Phys. B393, 413 (1993); S. Lukyanov, Comm. Math. Phys. 167, 183 (1995); S. Lukyanov, Mod. Phys. Lett. A12, 2911 (1997).

[14] A.B. Zamolodchikov, JETP Lett. 25, 468 (1977); H.-J. Thun, T.T. Truong, P.H. Weisz, Phys. Lett. B67, 321 (1977).

[15] J. Cardy and G. Mussardo, Nucl. Phys. B410, 451 (1993).

[16] G. Mussardo, preprint hep-th/940512.

[17] E. Jeckelmann, F. Gebhard and F. H. L. Essler, Phys. Rev. Lett. 85, 3910 (2000).

[18] D. Controzzi, F.H.L. Essler and A.M. Tsvelik, Phys. Rev. Lett. 86680 (2001).

[19] D. Controzzi, F.H.L. Essler and A.M. Tsvelik, in "New Theoretical Approaches to Strongly Correlated Systems", ed A.M. Tsvelik, NATO Science Series II Vol 23, preprint cond-mat/0011439.

[20] E. Jeckelmann, Phys. Rev. B 66, 045114 (2002).

[21] F.H.L. Essler, F. Gebhard and E. Jeckelmann, Phys. Rev. B 64, 5119 (2001).

[22] E. Jeckelmann, Phys. Rev. B 67, 075106 (2003).

[23] A. Schwartz, M. Dressel, G. Grüner, V. Vescoli, L. Degiorgi, T. Giamarchi, Phys. Rev. B 58, 1261 (1998); V. Vescoli, L. Degiorgi, W. Henderson, G. Grüner, K. P. Starkey, L. K. Montgomery, Science 281, 1181 (1998); W. Henderson, V. Vescoli, P. Tran, L. Degiorgi and G. Grüner, Eur. Phys. J. B 11, 365 (1999).

[24] M.G. Zacher, E. Arrigoni, W. Hanke and J.R. Schrieffer, Phys. Rev. B 57, 6370 (1998); D. Senechal, D. Perez and M. Pioro-Ladiere, Phys. Rev. Lett. 84, 522 (2000); R.N. Bannister and N. d'Ambrumenil, Phys. Rev. B 61, 4651 (2000).

[25] A. Parola and S. Sorella, Phys. Rev. B 57, 6444 (1998); A. Parola and S. Sorella, Phys. Rev. Lett. 76, 4604 (1996); K. Penc, K. Hallberg, F. Mila and H. Shiba, Phys. Rev. Lett. 77, 1390 (1996).

[26] P. B. Wiegmann, Sov. Sci. Rev. Ser. A, vol.2, p.41 (1980), Harwood Academic Publ., ed. I. M. Khalatnikov. 
[27] J. Voit, Eur. Phys. J. B 5, 505 (1998).

[28] O.A. Starykh, D.L. Maslov, W. Häusler, L.I. Glazman, in Low-Dimensional Systems, ed. T: Brandes, Lecture Notes in Physics, Springer (2000)

[29] F.H.L. Essler and A.M. Tsvelik, Phys. Rev. B 65, 115117 (2002).

[30] S. Lukyanov and A. B. Zamolodchikov, Nucl. Phys. B607, 437 (2001).

[31] F.H.L. Essler and A.M. Tsvelik, Phys. Rev. Lett. 90, 126401 (2003).

[32] F.H.L. Essler and A.M. Tsvelik, Phys. Rev. Lett. 88, 096403 (2002).

[33] A.A. Abrikosov, L.P. Gorkov and I.E. Dzyaloshinski, Methods of Quantum Field Theory in Statistical Physics, Dover (New York) 1975, page 168.

[34] X.G. Wen, Phys. Rev. B42, 6623 (1990); E. Arrigoni, Phys. Rev. B 61, 7909 (2000).

[35] D. Boies, C. Bourbonnais and A.-M. S. Tremblay, Phys. Rev. Lett. 74, 968 (1995).

[36] D.J. Scalapino, Y. Imry and P. Pincus, Phys. Rev. B11, 2042 (1975).

[37] S. Biermann, T. Giamarchi, A. Georges and A. Lichtenstein, Phys. Rev. Lett. 87, 276405 (2001).

[38] D. Controzzi and F.H.L. Essler, Phys. Rev. B 66, 165112 (2002).

Fabian H.L. Essler and Alexei M. Tsvelik

Department of Physics

Brookhaven National Laboratory

Upton, NY 11973-5000

USA 